OPEN ACCESS

Edited by:

Euripedes Constantino Miguel, University of São Paulo, Brazil

Reviewed by: Ewgeni Jakubovski, Hannover Medical School, Germany Aline S. Sampaio,

Universidade Federal da Bahia, Brazil

${ }^{*}$ Correspondence: Sónia Ferreira soniamgaf@gmail.com

Specialty section

This article was submitted to Mood and Anxiety Disorders, a section of the journal Frontiers in Psychiatry

Received: 13 August 2020 Accepted: 21 December 2020 Published: 20 January 2021

Citation:

Ferreira S, Couto B, Sousa M, Vieira R, Sousa N, Picó-Pérez M and Morgado P (2021) Stress Influences the Effect of Obsessive-Compulsive Symptoms on Emotion Regulation.

Front. Psychiatry 11:594541. doi: 10.3389/fpsyt.2020.594541

\section{Stress Influences the Effect of Obsessive-Compulsive Symptoms on Emotion Regulation}

\author{
Sónia Ferreira ${ }^{1,2,3 *}$, Beatriz Couto ${ }^{1,2,3}$, Mafalda Sousa ${ }^{1,2,3}$, Rita Vieira ${ }^{1,2,3}$, Nuno Sousa ${ }^{1,2,3}$, \\ Maria Picó-Pérez ${ }^{1,2,3}$ and Pedro Morgado ${ }^{1,2,3}$ \\ ${ }^{1}$ Life and Health Sciences Research Institute (ICVS), School of Medicine, University of Minho, Braga, Portugal, ${ }^{2}$ ICVS-3Bs PT \\ Government Associate Laboratory, Braga, Portugal, ${ }^{3}$ Clinical Academic Center-Braga (2CA), Braga, Portugal
}

Obsessive-compulsive disorder (OCD) is associated with emotion regulation impairments, namely the frequent use of maladaptive strategies such as suppression and the decreased use of reappraisal strategies. Additionally, these patients exhibit elevated stress levels. Since stress exposure affects emotion regulation abilities, stress might influence the relationship between obsessive-compulsive symptoms and emotion regulation. In this study, we explored the effects of stress and obsessive-compulsive symptoms on emotion regulation in a sample of healthy and OCD individuals. We used self-reported psychometric scales to measure stress levels, obsessive-compulsive symptoms, and emotion reappraisal and suppression skills. We applied multiple regression and mediation analyses. Our results demonstrated that increased reappraisal scores were associated with higher suppression scores. Additionally, elevated stress values predicted increased scores for suppression and decreased scores for reappraisal. Furthermore, the reappraisal abilities resulted from a combination of a direct effect of obsessive-compulsive symptoms and an indirect effect of obsessive-compulsive symptoms mediated by stress. The reliance on suppression strategies and the difficulty in using reappraisal approaches are explained by stress levels and are not directly explained by obsessive-compulsive symptoms. This study highlights the necessity of targeting stress in current therapy-based treatments for OCD.

Keywords: OCD, obsessive-compulsive disorder, PSS-10, ERQ, OCI-R, cognitive

\section{INTRODUCTION}

Obsessive-compulsive disorder (OCD) may arise from an interplay between genetic and environmental risk factors, namely exposure to stressful and traumatic life events $(1,2)$. Moreover, increases in general stress (e.g., job loss and family disease) and changes in routines throughout life are features associated with the development (3) and severity (4, 5 ) of OCD. OCD is characterized by elevated levels of anxiety and distress elicited by the presence of intrusive thoughts (obsessions) (6). The enhanced levels of distress might increase the hypothalamic-pituitary-adrenal (HPA) axis function resulting in an augmented stress response (7-9). In line with this assumption, previous studies have found correlations between perceived stress levels and obsessive-compulsive symptoms in OCD and healthy individuals (10-12), and between cortisol responses and obsessive-compulsive symptoms in healthy individuals (13). Additionally, other researchers demonstrated that increased cortisol 
levels are a hallmark of OCD also suggesting the hyperactivity of the HPA axis $(10,14)$, although contradictory findings were also found (11). Furthermore, brain anatomical and functional alterations in the striatum (caudate and putamen), hippocampus, amygdala, and medial and orbitofrontal prefrontal cortices have been reported for OCD and stress, suggesting that stress may exacerbate the bias toward habitual and ritualistic compulsive behaviors in OCD patients $(1,15-18)$.

OCD is also linked to emotional regulation deficits (1921). Past research has shown that OCD patients frequently suppress their emotions instead of using more beneficial reappraisal strategies (22-26). The constant use of suppression has counterproductive effects leading to more distress and intrusive thoughts $(27,28)$. Cognitive reappraisal consists of the alteration of the initial experiencing of affective stimuli. Two main strategies are commonly used for cognitive reappraisal: (1) reinterpretation-interpretation of the stimulus to achieve a more positive/pleasant connotation; (2) distancing-visualizing the stimulus from the perspective of an unrelated observer or an unreal situation. For example, OCD patients can reinterpret their intrusive thoughts as a common event that occurs in everyone's life. Additionally, OCD patients can distance themselves from the consequences of their intrusive thoughts by thinking of them as not real. Expressive suppression relies on the inhibition of behavioral and emotional responses in the presence of affective stimuli (29-32). The emotional appraisal and regulation processes are linked to stress mechanisms. Acute and social stressors lead to the engagement of maladaptive emotion regulation strategies such as worry and rumination (33). Thus, the chronic use of these strategies might in turn augment the stress response. Indeed, maladaptive emotion regulation strategies have been associated with increased stress responses $(33,34)$, while reappraisal leads to enhanced stress recovery (34) in healthy participants and individuals with anxiety disorders. A recent meta-analysis also reported that reappraisal of fear/negative emotions induced by stressful tasks decreases the heart rate in healthy individuals (35). Moreover, emotion regulation difficulties translate into decreased heart rate variability $(36,37)$, a well-known biomarker of stress (38). Lastly, diminished cortisol and perceived stress levels in response to an acute stressor were observed after cognitivebehavioral stress management (39). These authors reported that the alterations in stress response were associated with changes in emotion appraisal.

In this way, stress may play a significant role in the relationship between OCD and emotion regulation. In this study, we aim to investigate the impact of stress and obsessivecompulsive (OC) symptoms on emotion regulation in a sample of non-psychiatric and OCD individuals using psychometric instruments. Based on the previous literature, we assume that higher scores for stress and OC symptoms are associated with less effective use of emotion reappraisal and enhanced use of emotion

Abbreviations: OCD, obsessive-compulsive disorder; HPA, hypothalamicpituitary-adrenal; OC, obsessive-compulsive; Y-BOCS, Yale-Brown Obsessive Compulsive Scale; ERQ, Emotion Regulation Questionnaire; OCI-R, ObsessiveCompulsive Inventory-Revised; PSS-10, 10-items Perceived Stress Scale. suppression strategies. Furthermore, past evidence suggests a link between stress and OC symptoms. Thus, we hypothesize that stress mediates the effect of $\mathrm{OC}$ symptoms on emotion regulation. This study elucidates the role of stress on OCD providing new recommendations for current psychotherapy approaches.

\section{MATERIALS AND METHODS \\ Participants}

We included OCD patients and non-psychiatric control participants in this study. These groups of participants with low and high OC symptoms were recruited to have a wider range of OC symptomatology. OCD patients were recruited at the Psychiatry Unit of Hospital de Braga (Braga, Portugal) and diagnosed by a psychiatrist (PM) according to the Diagnostic and Statistical Manual of Mental Disorders (DSM-5) criteria. The patients were under treatment as usual or were treatment naïve. We excluded patients with comorbid psychiatric disorders or a history of neurological disorders. The control participants were recruited among the local community according to the age, gender, and education level of the patients, did not have a history of psychiatric/neurological disorders, and were not taking psychiatric medication.

All participants signed an informed consent. The study was approved by the ethics committees of Hospital de Braga (Comissão de Ética para a Saúde), Braga, Portugal, and University of Minho (Subcomissão de Ética para as Ciências da Vida e da Saúde), Braga, Portugal, and respected the Declaration of Helsinki principles.

\section{Psychometric Evaluation}

The Yale-Brown Obsessive Compulsive Scale (Y-BOCS) was applied to evaluate the disease severity in OCD patients (4042). We also applied the Emotion Regulation Questionnaire (ERQ) to measure reappraisal and suppression abilities $(43,44)$. The Obsessive-Compulsive Inventory-Revised (OCIR) was also used to measure OCD severity and dimensions (washing, checking, ordering, hoarding, obsessing, and neutralizing subscales) $(45,46)$. The 10 -items Perceived Stress Scale (PSS-10) was also applied to quantify selfperceived stress levels $(10,47,48)$. PSS-10 measures prolonged psychological stress experienced in the month preceding the scale application.

\section{Statistical Analysis}

The statistical analysis was performed with JASP (version 0.11.1; JASP Team, University of Amsterdam, The Netherlands). Pvalues under 0.05 were considered statistically significant. All statistical tests were two-tailed. The assumption of normality was assessed with the Shapiro-Wilk test. The Cohen's $d$ effect size was calculated for all results: $0.2 \leq d<0.5$ small effect; $0.5 \leq d<0.8$ medium effect; $d \geq 0.8$ large effect (49).

First, we evaluated differences in demographic (age, gender, and education) and psychometric (ERQ reappraisal and suppression, PSS-10, and OCI-R total and subscales) variables between the OCD and control group using independent 
samples $t$-tests for parametric variables and the Mann-Whitney $[U]$ test for non-parametric variables (the chi-squared test was used for the categorical variable gender $\left.\left[\chi^{2}\right]\right)$. We used Bonferroni correction for multiple comparisons in the OCI-R subscales (washing, checking, ordering, hoarding, obsessing, and neutralizing).

Moreover, we explored the association among the variables (age, education, ERQ reappraisal and suppression, PSS-10, and OCI-R total) for all participants and within each group (OCD and control) using Pearson's or Spearman's correlation, depending on the normality of the variables. We used Bonferroni correction to correct for multiple comparisons.

After, we used two multiple regression models to study which demographic and psychometric variables predicted the ERQ reappraisal and ERQ suppression scores in the total sample. We tested the following predictors: age, gender, education, PSS10, OCI-R total, and ERQ reappraisal/ERQ suppression. The assumptions of normality, linearity, and homoscedasticity were verified by visual inspection of the Q-Q and residuals-predicted plots. Correlations between residuals and multicollinearity were verified with the Durbin-Watson value (between 1.5 and 2.5) and tolerance $(>0.1)$ and variance inflation factor $(<10)$ values $(50,51)$.
Lastly, we performed a mediation analysis to understand if the OCI-R total score (predictor variable) predicted the ERQ reappraisal and suppression scores (outcome variables) when mediated by the PSS-10 score (mediator variable), using age, gender, and education as background confounders. We followed the assumptions for mediation analyses defined by Kenny and colleagues (52-54): the predictor variable influences the mediator variable; the mediator variable affects the outcome variable when controlling for the predictor variable. The influence of the predictor variable on the outcome variable is no longer a requirement for mediation analysis according to these authors. We applied the bias-corrected percentile bootstrap method with 1,000 replications. The use of bootstrapping in mediation analysis consists of a non-parametric method to estimate the sampling distribution of indirect effects without prior assumptions of the distribution shape, providing higher statistical power and more accurate estimation of confidence intervals than standard methods (55-57). This analysis was performed using the total sample. We assessed direct, indirect, and total effects of the OCI$\mathrm{R}$ score on the ERQ reappraisal/suppression score. The indirect effect represents the amount of mediation by the PSS-10 score and the total effect result from the sum of direct and indirect effects $(57,58)$.

TABLE 1 | Description of the demographic and psychometric variables for the obsessive-compulsive and control group and for the whole sample, and representation of the statistical differences between groups (independent samples $t$-test, Mann-Whitney test [U], and chi-squared test [ $\left.X^{2}\right]$; $p_{\text {bonf }}-p$-value after Bonferroni correction; d-Cohen's effect size).

\begin{tabular}{|c|c|c|c|c|}
\hline & $\begin{array}{c}\text { OCD } \\
(n=42)\end{array}$ & $\begin{array}{l}\text { Control } \\
(n=22)\end{array}$ & $\begin{array}{l}\text { Total sample } \\
\qquad(n=64)\end{array}$ & $\begin{array}{l}\text { Statistical results between groups } \\
\text { (OCD vs. control) }\end{array}$ \\
\hline Age (years) & $27.0(13.7)$ & $24.0(15.2)$ & $27.0(15.2)$ & $U=392.00 ; p=0.325 ; d=0.25$ \\
\hline Gender (F | M) & $27 \mid 15$ & $13 \mid 9$ & $40 \mid 24$ & $X_{(1)}^{2}=0.17 ; p=0.683 ; d=0.10$ \\
\hline Education (years) & $13.5(4.7)$ & $13.0(3.7)$ & $13.5(4.2)$ & $U=431.50 ; p=0.668 ; d=0.11$ \\
\hline \multicolumn{5}{|l|}{$\mathrm{Y}_{\mathrm{BOCS}}^{\dagger}$} \\
\hline Total & $28.0(5.0)$ & - & - & - \\
\hline Obsessions & $13.0(5.0)$ & - & - & - \\
\hline Compulsions & $14.0(3.0)$ & - & - & - \\
\hline \multicolumn{5}{|l|}{$\mathrm{ERQ}$} \\
\hline Reappraisal & $24.9 \pm 9.5$ & $30.1 \pm 7.9$ & $29.0(12.5)$ & $t_{(62)}=-2.19 ; p=0.032 ; d=-0.58^{\star}$ \\
\hline Suppression & $14.3 \pm 5.1$ & $15.0 \pm 5.9$ & $14.5 \pm 5.4$ & $t_{(62)}=-0.53 ; p=0.595 ; d=-0.14$ \\
\hline PSS-10 & $22.3 \pm 8.0$ & $15.4 \pm 7.2$ & $19.9 \pm 8.3$ & $t_{(62)}=3.40 ; p=0.001 ; d=0.89^{\star}$ \\
\hline \multicolumn{5}{|l|}{ OCl-R } \\
\hline Total & $31.8 \pm 14.0$ & $15.4 \pm 10.3$ & $26.2 \pm 15.0$ & $t_{(62)}=4.82 ; p=9.777 \times 10^{-6} ; d=1.27^{*}$ \\
\hline Washing & $4.5(6.0)$ & $1.0(1.7)$ & $2.0(5.2)$ & $\begin{array}{c}U=175.50 ; p_{\text {bonf }}=2.654 \times 10^{-4} \\
d=1.17^{\star}\end{array}$ \\
\hline Checking & $4.0(6.0)$ & $1.5(2.0)$ & $3.0(4.5)$ & $\begin{array}{c}U=190.00 ; p_{\text {bonf }}=7.140 \times 10^{-4} \\
d=1.10^{\star}\end{array}$ \\
\hline Ordering & $5.0(5.0)$ & $4.0(4.0)$ & $4.0(5.2)$ & $U=349.00 ; p_{\text {bonf }}=0.660 ; d=0.41$ \\
\hline Hoarding & $3.0(4.0)$ & $3.0(3.5)$ & $3.0(4.0)$ & $U=464.50 ; p_{\text {bonf }}=1.000 ; d=0.01$ \\
\hline Obsessing & $8.0(6.0)$ & $2.5(4.5)$ & $5.5(7.0)$ & $\begin{array}{c}U=135.00 ; p_{\text {bonf }}=2.128 \times 10^{-5} \\
d=1.42^{\star}\end{array}$ \\
\hline Neutralizing & $3.0(7.0)$ & $1.0(2.0)$ & $2.0(6.0)$ & $U=292.00 ; p_{\text {bonf }}=0.090 ; d=0.63$ \\
\hline
\end{tabular}

Data represents mean \pm standard deviation for normally distributed variables and median (interquartile range) for the other variables; OCD, obsessive-compulsive disorder; $F$, female;

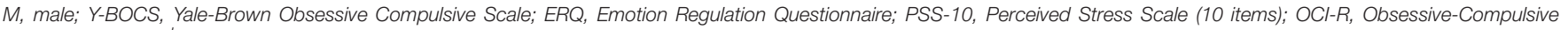
Inventory-Revised; ${ }^{\dagger}$ Four patients with missing data; ${ }^{*}$ Statistically significant differences between groups. 
TABLE 2 | Results of Pearson's (rp; normally distributed variables) and Spearman's (rs; variables not normally distributed) correlation among demographic and psychometric variables for the complete sample (p bonf - $p$-value after Bonferroni correction; $d$-Cohen's effect size).

\begin{tabular}{|c|c|c|c|c|c|}
\hline & Education (years) & ERQ reappraisal & ERQ suppression & PSS-10 & OCl-R total \\
\hline Age (years) & $\begin{array}{c}r s=-0.14 \\
p_{\text {bonf }}=1.000 \\
p=0.254 \\
d=-0.28\end{array}$ & $\begin{array}{c}r s=6.31 \times 10^{-4} \\
p_{\text {bonf }}=1.000 \\
p=0.996 \\
d=1.3 \times 10^{-3}\end{array}$ & $\begin{array}{c}r s=0.17 \\
p_{\text {bonf }}=1.000 \\
p=0.187 \\
d=0.34\end{array}$ & $\begin{array}{c}r s=0.18 \\
p_{\text {bonf }}=1.000 \\
p=0.151 \\
d=0.37\end{array}$ & $\begin{array}{c}r s=0.17 \\
p_{\text {bonf }}=1.000 \\
p=0.172 \\
d=0.34\end{array}$ \\
\hline Education (years) & - & $\begin{array}{c}r s=-0.10 \\
p_{\text {bonf }}=1.000 \\
p=0.435 \\
d=-0.20\end{array}$ & $\begin{array}{c}r s=-0.18 \\
p_{\text {bonf }}=1.000 \\
p=0.145 \\
d=-0.37\end{array}$ & $\begin{array}{c}r s=0.13 \\
p_{\text {bonf }}=1.000 \\
p=0.301 \\
d=0.26\end{array}$ & $\begin{array}{c}r s=-0.04 \\
p_{\text {bonf }}=1.000 \\
p=0.729 \\
d=-0.08\end{array}$ \\
\hline ERQ reappraisal & - & - & $\begin{array}{c}r s=0.32 \\
p_{\text {bonf }}=0.150 \\
p=0.010 \\
d=0.67\end{array}$ & $\begin{array}{c}r s=-0.30 \\
p_{\text {bonf }}=0.255 \\
p=0.017 \\
d=-0.63\end{array}$ & $\begin{array}{c}r s=-0.23 \\
p_{\text {bonf }}=0.945 \\
p=0.063 \\
d=-0.47\end{array}$ \\
\hline ERQ suppression & - & - & - & $\begin{array}{c}r p=0.06 \\
p_{\text {bonf }}=1.000 \\
p=0.636 \\
d=0.12\end{array}$ & $\begin{array}{c}r p=-0.03 \\
p_{\text {bonf }}=1.000 \\
p=0.789 \\
d=-0.06\end{array}$ \\
\hline PSS-10 & - & - & - & - & $\begin{array}{c}r p=0.62 \\
p_{\text {bonf }}=7.815 \times 10^{-7 *} \\
p=5.210 \times 10^{-8} ; \\
d=1.58\end{array}$ \\
\hline
\end{tabular}

ERQ, Emotion Regulation Questionnaire; PSS-10, Perceived Stress Scale (10 items); OCI-R, Obsessive-Compulsive Inventory-Revised; *Statistically significant correlations.

\section{RESULTS}

We included 43 OCD patients and 22 control participants. One OCD patient was excluded because he/she did not fill the OCI-R scale. Three patients were treatment naïve, 3 patients were not under medication, and the other patients were taking psychotropic medication (clomipramine, fluoxetine, fluvoxamine, sertraline, or escitalopram). Nine patients were being treated with psychotherapy (13 patients with missing information).

Table 1 contains the descriptive and statistical values for demographic and psychometric data. OCD and control groups were not different in terms of age, gender ratio, and education level. Additionally, we observed statistically significant increases in the PSS-10 score, and the OCI-R total, washing, checking, and obsessing scores in the OCD group. Moreover, OCD participants had decreased scores for the ERQ reappraisal subscale.

Table 2 summarizes Pearson's and Spearman's correlation results for the complete sample. We observed a positive correlation between the OCI-R and PSS-10 scores. Within the OCD group, we found a positive correlation between the OCI$\mathrm{R}$ and PSS-10 scores (Supplementary Table 1). For the control group, we did not detect significant correlations after correcting for multiple comparisons (Supplementary Table 2). However, the correlation between the OCI-R and PSS-10 scores was also statistically significant in the control groups with the uncorrected p-value (Supplementary Table 2).

The regression model for the ERQ reappraisal score yielded statistical significance $\left[F_{(6,56)}=3.53 ; p=0.005 ; R^{2}=0.27\right]$. The ERQ reappraisal score was significantly predicted by gender (beta \pm standard error $=6.18 \pm 2.49 ; t=2.48, p=0.016$, standardized beta $=0.33$; effect size $d=0.81$ ), the ERQ suppression score $(0.76 \pm 0.22 ; t=3.47, p=0.001,0.44$; $d=1.14)$, and the PSS-10 score $(-0.42 \pm 0.17 ; t=-2.40$, $p=0.020,-0.38 ; d=-0.96)$. The regression model for the ERQ suppression score was also statistically significant $\left[F_{(6,56)}=4.94 ; p=4.000 \times 10^{-4} ; R^{2}=0.35\right]$. The ERQ suppression score was significantly predicted by gender $(-4.93$ $\left.\pm 1.30 ; t=-3.78, p=4.000 \times 10^{-4},-0.45 ; d=-1.14\right)$, the ERQ reappraisal score $(0.23 \pm 0.07 ; t=3.47, p=0.001$, $0.40 ; d=1.01)$, and the PSS-10 score $(0.24 \pm 0.10 ; t=2.56$, $p=0.013,0.38 ; d=0.96$ ). Figure 1 represents the results of both regression models. In conclusion, increased values of ERQ reappraisal were associated with higher ERQ suppression scores. Female participants had higher values in ERQ reappraisal and lower values in ERQ suppression. Elevated values of PSS10 corresponded to increased scores in ERQ suppression and decreased ERQ reappraisal scores.

For the mediation analysis, the direct effect of OCI-R on ERQ reappraisal (beta \pm standard error $=-0.06 \pm 0.09$, $p=0.502 ; d=-0.17)$ and suppression $(-0.06 \pm 0.05, p=0.217$; $d=-0.31$ ) was not statistically significant. Moreover, the indirect effect of OCI-R on ERQ reappraisal $(-0.09 \pm 0.06$, $p=0.116 ; d=-0.40)$ and suppression $(0.06 \pm 0.03, p=0.075$; $d=0.46)$ when mediated by PSS-10 was also not statistically significant. Nonetheless, the total effect (combination of direct and indirect effects) was statistically significant for the ERQ reappraisal $(-0.16 \pm 0.08, p=0.036 ; d=-0.54)$ but not for the ERQ suppression score $\left(-4.00 \times 10^{-3} \pm 0.04, p=0.916 ; d\right.$ $=-0.03)$. Moreover, the ERQ reappraisal and suppression score had a statistically significant association $(16.95 \pm 5.55, p=0.002$; $d=0.83$ ) (57). Figure 2 represents the mediation analysis results. 


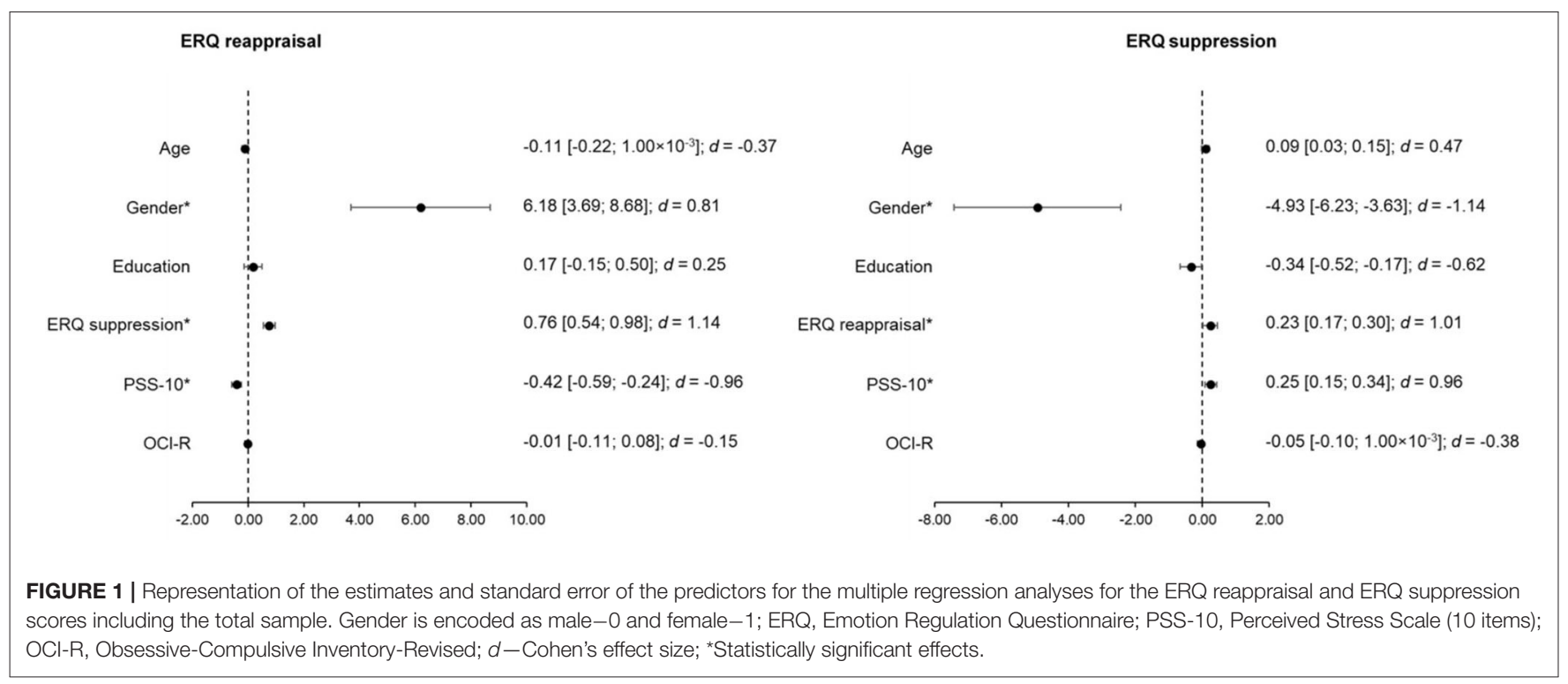

In conclusion, the ERQ reappraisal score is explained by the direct effect of the OCI-R score combined with the OCI-R effect mediated by the PSS-10 score. Moreover, the ERQ reappraisal and suppression score influence each other.

\section{DISCUSSION}

In this study, we evaluated if stress and OC symptoms have a negative effect on emotion regulation measures in a sample composed of OCD and healthy participants. Our main results demonstrated that suppression and reappraisal abilities are predicted by gender and stress levels but not by OC symptoms. Moreover, we observed that the reappraisal score results from a combination of a direct effect of OC symptoms and an indirect effect of OC symptoms mediated by stress levels.

First, our results showed that OCD patients had reduced reappraisal scores in line with past findings (21-26). However, in contrast with these findings, we did not observe an augmented use of suppression in the OCD group. Most of the patients were under pharmacological treatment. Thus, they might have reduced the use of suppression to attenuate the emotional impact of obsessions and distress. However, the median YBOCS score indicates severe to extreme OC symptomatology despite the treatment. Moreover, some authors did not find increased suppression scores $(21,24,26)$ even in OCD patients without medication. In this way, other factors may affect the suppression score in OCD individuals. On the other hand, the control participants included in this study may regularly use suppression strategies given the higher difficulty and cognitive cost in using reappraisal for emotion regulation (59-61). In agreement with this, we found that higher emotion reappraisal abilities were predicted by increased suppression scores and vice versa. Furthermore, there was a positive influence between reappraisal and suppression scores in the mediation analysis. Thus, our results might indicate that effective emotion regulation depends on the use of both strategies. Indeed, past findings showed that the frequent practice of reappraisal is not linked to reduced use of suppression strategies (62). Additionally, studies exploring the spontaneous use of emotion regulation strategies showed that reappraisal is not applied more often than suppression (63).

We also found augmented levels of perceived stress in the OCD group supporting the interplay between OCD and stress $(1,10,14,64)$. This outcome was further reinforced by a strong positive correlation between stress and OC scores in the total sample and the OCD group.

Both the suppression and reappraisal scores were predicted by gender and stress levels but not by the OC score. Moreover, the reappraisal score resulted from a combination of a direct effect of OC symptoms and an indirect effect of these symptoms mediated by perceived stress levels. Past researchers also reported that women express more their emotions and have more practice at successfully regulating them $(29,65)$, while men are culturally shaped to suppress some type of emotions (e.g., sadness and fear) (66). Thus, males might have more difficulties in identifying, accepting, and regulating emotions. Moreover, women use suppression strategies less frequently than men (67). Consistent with our findings, previous researchers also found that maladaptive strategies (suppression and rumination) and reappraisal were positively and negatively associated with stress symptoms, respectively $(62,68)$. Also, individuals under stressful conditions are more predisposed to the effects of negative emotional stimuli (69-71), and are ineffective in distracting themselves $(69,72)$ or reappraising their emotions $(71,73)$ when exposed to affective stimuli. Moreover, stress leads to the engagement of maladaptive strategies such as worry and rumination (33). Thus, individuals under stress may be more prone to use suppressing strategies. These findings may result from stressinduced impairment of cognitive processes (e.g., cognitive flexibility and inhibitory and goal-directed behavior) due 


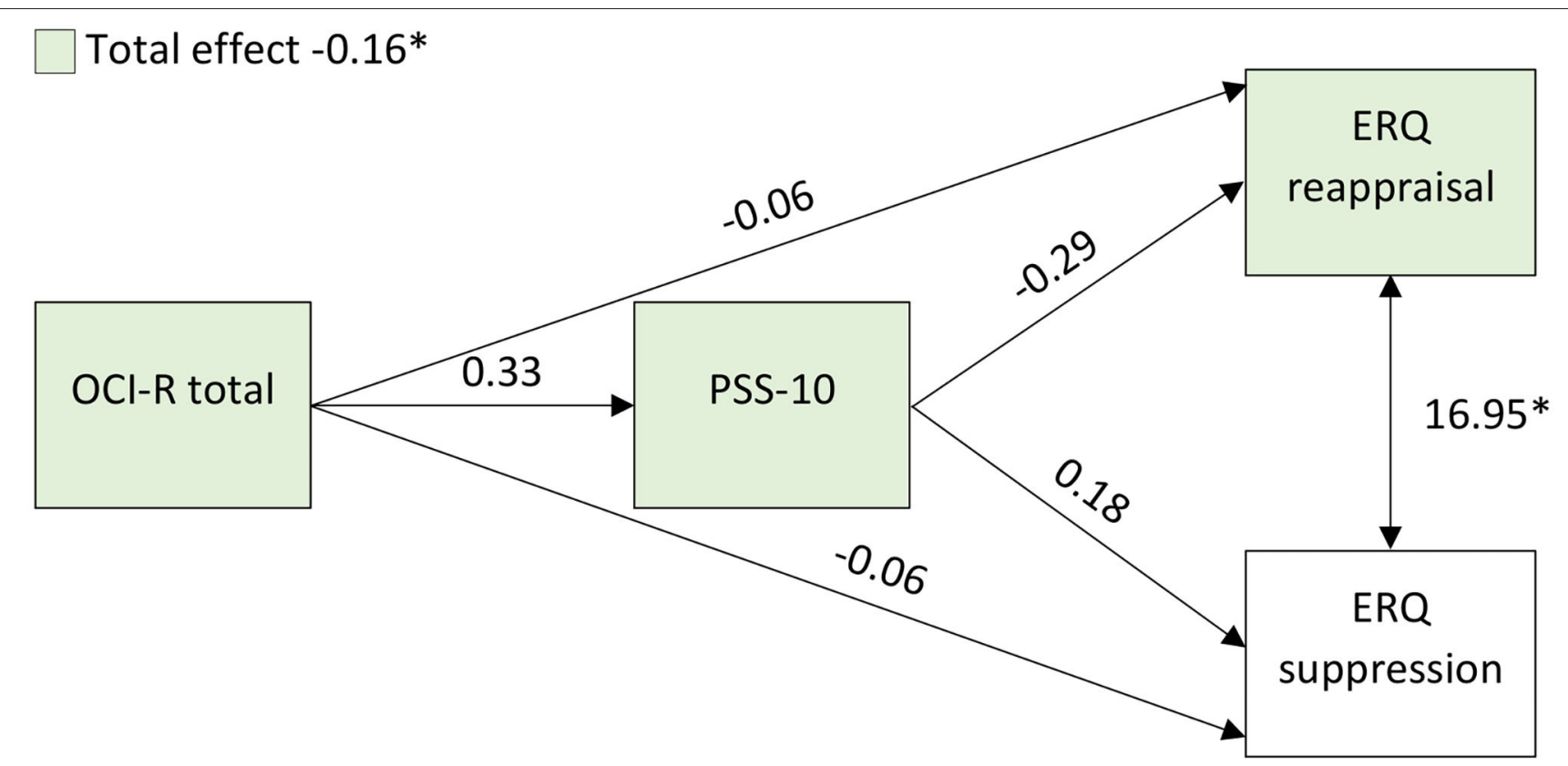

FIGURE 2 | Representation of the mediation analysis results. The values represent the estimates. ERQ, Emotion Regulation Questionnaire; PSS-10, Perceived Stress Scale (10 items); OCl-R, Obsessive-Compulsive Inventory-Revised; *Statistically significant effects.

to the disruption of prefrontal function (74). Thus, stress might inhibit the prefrontal cortical activity hampering the modulation of limbic regions (e.g., amygdala) during emotion regulation $(18,70)$. Indeed, these brain regions are also implicated in emotion regulation processes $(32,75)$. In summary, OCD individuals have elevated stress symptoms that might weaken their ability to use emotion reappraisal strategies. Their cognitive resources are impaired by stress leading to an increased response to negative emotions (59). Instead of reappraisal, they may choose more effortless maladaptive strategies (e.g., suppression and compulsions) (61) to regulate their emotions, leading to a rebound effect on distress and anxiety levels $(27,28,31,68,76)$.

Our findings are limited by the lack of control for anxiety and depression levels. Both OC and stress symptoms are associated with anxious and depressed mood (77). Yap et al. (78) found that OCD severity was not associated with emotion regulation deficits when controlling for anxiety and depression scores. Moreover, Moore et al. (62) found associations between the ERQ scores and anxiety and depression symptoms. Thus, anxiety and depression might have a significant impact on emotional regulation $(79,80)$. Our results might have also been affected by the fact that most of the OCD patients selected for this study were medicated and some were frequenting psychotherapy sessions. Moreover, our study has a cross-sectional design hampering the analysis of stress and OC symptoms variations on emotional regulation. Future studies with cognitive-behavioral therapy for OCD and stress management might provide further insights. Additionally, our sample had a higher proportion of female individuals. However, the main conclusions were controlled for gender ratio. Finally, our results need to be replicated with larger samples to increase the study power. Indeed, some of our findings did not show a large effect size (differences between OCD and control groups in the reappraisal score, and the total effect of OC symptoms in the reappraisal score in the mediation analysis).

\section{CONCLUSION}

This study provides a novel perspective of emotional regulation impairments in OCD. The reliance on suppression strategies and the difficulty in using reappraisal approaches are explained by stress levels and not directly explained by OC symptoms. Our conclusions support the inclusion of stress management in cognitive-behavioral therapy treatments to improve the processes of emotion regulation in OCD patients.

\section{DATA AVAILABILITY STATEMENT}

The raw data supporting the conclusions of this article will be made available by the authors, without undue reservation.

\section{ETHICS STATEMENT}

The studies involving human participants were reviewed and approved by Comissão de Ética para a Saúde, Hospital de Braga and Subcomissão de Ética para as Ciências da Vida e da Saúde, University of Minho. The patients/participants provided their written informed consent to participate in this study. 


\section{AUTHOR CONTRIBUTIONS}

SF, BC, MS, RV, and MP-P: study design, data collection, data analysis, and manuscript writing. NS, MP-P, and PM: study design, manuscript writing, and study supervision. All authors contributed to the article and approved the submitted version.

\section{FUNDING}

This work has been funded by National funds, through the Foundation for Science and Technology (FCT) - project UIDB/50026/2020 and UIDP/50026/2020; and by the projects NORTE-01-0145-FEDER-000013 and NORTE-01-0145-FEDER000023, supported by Norte Portugal Regional Operational Programme (NORTE 2020), under the PORTUGAL 2020 Partnership Agreement, through the European Regional

\section{REFERENCES}

1. Adams TG, Kelmendi B, Brake CA, Gruner P, Badour CL, Pittenger C. The role of stress in the pathogenesis and maintenance of obsessive-compulsive disorder. Chronic Stress. (2018) 2:247054701875804. doi: 10.1177/2470547018758043

2. Brander G, Pérez-Vigil A, Larsson H, Mataix-Cols D. Systematic review of environmental risk factors for Obsessive-Compulsive Disorder: a proposed roadmap from association to causation. Neurosci Biobehav Rev. (2016) 65:3662. doi: 10.1016/j.neubiorev.2016.03.011

3. Coles ME, Johnson EM, Schubert JR. Retrospective reports of the development of obsessive compulsive disorder: extending knowledge of the protracted symptom phase. Behav Cogn Psychother. (2011) 39:57989. doi: 10.1017/S135246581100004X

4. Lin H, Katsovich L, Ghebremichael M, Findley DB, Grantz H, Lombroso PJ, et al. Psychosocial stress predicts future symptom severities in children and adolescents with Tourette syndrome and/or obsessivecompulsive disorder. J Child Psychol Psychiatry Allied Discip. (2007) 48:15766. doi: 10.1111/j.1469-7610.2006.01687.x

5. Kracker Imthon A, Antônio Caldart C, do Rosário MC, Fontenelle LF, Constantino Miguel E, Arzeno Ferrão Y. Stressful life events and the clinical expression of Obsessive-Compulsive Disorder (OCD): an exploratory study. J Clin Med. (2020) 9:3371. doi: 10.3390/jcm9103371

6. American Psychiatric Association. Diagnostic Statistical Manual of Mental Disorders (DSM-5). 5th ed. American Psychiatric Association Pub, 2013 (2013).

7. Kathol RG, Noyes R, Lopez A. Similarities in hypothalamic-pituitaryadrenal axis activity between patients with panic disorder and those experiencing external stress. Psychiatr Clin North Am. (1988) 11:33548. doi: 10.1016/S0193-953X(18)30501-X

8. de Kloet CS, Vermetten E, Geuze E, Kavelaars A, Heijnen CJ, Westenberg HGM. Assessment of HPA-axis function in posttraumatic stress disorder: pharmacological and non-pharmacological challenge tests, a review. J Psychiatr Res. (2006) 40:550-67. doi: 10.1016/j.jpsychires.2005.08.002

9. Faravelli C. Childhood stressful events, HPA axis and anxiety disorders. World J Psychiatry. (2012) 2:13. doi: 10.5498/wjp.v2.i1.13

10. Morgado P, Freitas D, Bessa JM, Sousa N, Cerqueira JJ. Perceived stress in obsessive-compulsive disorder is related with obsessive but not compulsive symptoms. Front Psychiatry. (2013) 4:21. doi: 10.3389/fpsyt. 2013.00021

11. Koumantarou Malisiova E, Mourikis I, Chalimourdas T, Nianiakas N, Michou M, Mantzou A, et al. Low hair cortisol concentrations in obsessive compulsive disorder: a cross-sectional study. $J$ Psychiatr Res. (2020) 131:187-93. doi: 10.1016/j.jpsychires.2020. 09.014
Development Fund (ERDF). This work was additionally supported by a research grant from the Clinical Academic Center, Braga, Portugal (Treating refractory obsessivecompulsive disorder using real-time functional magnetic resonance imaging neurofeedback). SF was supported by a combined Ph.D. scholarship from the FCT and the company iCognitus4ALL - IT Solutions, Lda (PD/BDE/127839/2016). MP-P was supported by an institutional post-doctoral grant (EMED_BPD_2019_002). RV was supported by an FCT research fellowship (UMINHO/BI/340/2018).

\section{SUPPLEMENTARY MATERIAL}

The Supplementary Material for this article can be found online at: https://www.frontiersin.org/articles/10.3389/fpsyt. 2020.594541/full\#supplementary-material
12. Ashraf F, Jibeen T, Masood A. Perceived stress in relation to obsessions and compulsions in south asian adults: moderating role of sociodemographic characteristics. Community Ment Health J. (2020) 56:68091. doi: 10.1007/s10597-019-00529-y

13. Melia CS, Soria V, Salvat-Pujol N, Cabezas Á, Nadal R, Urretavizcaya M, et al. Sex-specific association between the cortisol awakening response and obsessive-compulsive symptoms in healthy individuals. Biol Sex Differ. (2019) 10:55. doi: 10.1186/s13293-019-0273-3

14. Sousa-Lima J, Moreira PS, Raposo-Lima C, Sousa N, Morgado P. Relationship between obsessive compulsive disorder and cortisol: systematic review and meta-analysis. Eur Neuropsychopharmacol. (2019) 29:118598. doi: 10.1016/j.euroneuro.2019.09.001

15. Picó-Pérez M, Moreira PS, Ferreira VDM, Radua J, Mataix-Cols D, Sousa N, et al. Modality-specific overlaps in brain structure and function in Obsessivecompulsive disorder: multimodal meta-analysis of case-control MRI studies. Neurosci Biobehav Rev. (2020) doi: 10.1016/j.neubiorev.2020.01.033

16. Li Q, Yan J, Liao J, Zhang X, Liu L, Fu X, et al. Distinct effects of social stress on working memory in obsessive-compulsive disorder. Neurosci Bull. (2020) doi: 10.1007/s12264-020-00579-3. [Epub ahead of print].

17. Dedovic K, D'Aguiar C, Pruessner JC. What stress does to your brain: a review of neuroimaging studies. Can J Psychiatry. (2009) 54:615. doi: 10.1177/070674370905400104

18. Godoy LD, Rossignoli MT, Delfino-Pereira P, Garcia-Cairasco N, de Lima Umeoka EH. A Comprehensive overview on stress neurobiology: basic concepts and clinical implications. Front Behav Neurosci. (2018) 12:123. doi: 10.3389/fnbeh.2018.00127

19. Goldberg X, Cardoner N, Alonso P, López-Solà C, Real E, HernándezRibas $\mathrm{R}$, et al. Inter-individual variability in emotion regulation: pathways to obsessive-compulsive symptoms. J Obsessive Compuls Relat Disord. (2016) 11:105-12. doi: 10.1016/j.jocrd.2016.10.002

20. Zilverstand A, Parvaz MA, Goldstein RZ. Neuroimaging cognitive reappraisal in clinical populations to define neural targets for enhancing emotion regulation. A systematic review. Neuroimage. (2017) 151:105-16. doi: 10.1016/j.neuroimage.2016.06.009

21. Thorsen ALL, de Wit SJJ, de Vries FEE, Cath DCC, Veltman DJJ, van der Werf YDD, et al. Emotion regulation in obsessive-compulsive disorder, unaffected siblings, and unrelated healthy control participants. Biol Psychiatry Cogn Neurosci Neuroimaging. (2019) 4:352-60. doi: 10.1016/j.bpsc.2018.03.007

22. Fernández de la Cruz L, Landau D, Iervolino AC, Santo S, Pertusa A, Singh $\mathrm{S}$, et al. Experiential avoidance and emotion regulation difficulties in hoarding disorder. J Anxiety Disord. (2013) 27:204-9. doi: 10.1016/j.janxdis.2013.01.004

23. Yazici KU, Yazici IP. Decreased theory of mind skills, increased emotion dysregulation and insight levels in adolescents diagnosed with obsessive compulsive disorder. Nord J Psychiatry. (2019) 73:462-9. doi: 10.1080/08039488.2019.1652341 
24. De Wit SJ, Van Der Werf YD, Mataix-Cols D, Trujillo JP, Van Oppen P, Veltman DJ, et al. Emotion regulation before and after transcranial magnetic stimulation in obsessive compulsive disorder. Psychol Med. (2015) 45:3059-73. doi: 10.1017/S0033291715001026

25. Fink J, Pflugradt E, Stierle C, Exner C. Changing disgust through imagery rescripting and cognitive reappraisal in contaminationbased obsessive-compulsive disorder. J Anxiety Disord. (2018) 54:36-48. doi: 10.1016/j.janxdis.2018.01.002

26. Paul S, Simon D, Endrass T, Kathmann N. Altered emotion regulation in obsessive-compulsive disorder as evidenced by the late positive potential. Psychol Med. (2016) 46:137-47. doi: 10.1017/S0033291715 001610

27. Janeck AS, Calamari JE. Thought suppression in obsessive-compulsive disorder. Cognit Ther Res. (1999) 23:497-509. doi: 10.1023/A:1018720404750

28. Najmi S, Riemann BC, Wegner DM. Managing unwanted intrusive thoughts in obsessive-compulsive disorder: relative effectiveness of suppression, focused distraction, and acceptance. Behav Res Ther. (2009) 47:494503. doi: 10.1016/j.brat.2009.02.015

29. Webb TL, Miles E, Sheeran P. Dealing with feeling: a meta-analysis of the effectiveness of strategies derived from the process model of emotion regulation. Psychol Bull. (2012) 138:775-808. doi: 10.1037/a0027600

30. Dörfel D, Lamke J-P, Hummel F, Wagner U, Erk S, Walter H. Common and differential neural networks of emotion regulation by detachment, reinterpretation, distraction, and expressive suppression: a comparative fMRI investigation. Neuroimage. (2014) 101:298-309. doi: 10.1016/j.neuroimage.2014.06.051

31. Naragon-Gainey K, McMahon TP, Chacko TP. The structure of common emotion regulation strategies: a meta-analytic examination. Psychol Bull. (2017) 143:384-427. doi: 10.1037/bul0000093

32. Buhle JT, Silvers JA, Wager TD, Lopez R, Onyemekwu C, Kober H, et al. Cognitive reappraisal of emotion: a meta-analysis of human neuroimaging studies. Cereb Cortex. (2014) 24:2981-90. doi: 10.1093/cercor/bht154

33. Denson TF, Spanovic M, Miller N. Cognitive appraisals and emotions predict cortisol and immune responses: a meta-analysis of acute laboratory social stressors and emotion inductions. Psychol Bull. (2009) 135:82353. doi: 10.1037/a0016909

34. Lewis EJ, Yoon KL, Joormann J. Emotion regulation and biological stress responding: associations with worry, rumination, and reappraisal. Cogn Emot. (2018) 32:1487-98. doi: 10.1080/02699931.2017.1310088

35. Zaehringer J, Jennen-Steinmetz C, Schmahl C, Ende G, Paret C. Psychophysiological effects of downregulating negative emotions: insights from a meta-analysis of healthy adults. Front Psychol. (2020) 11:470. doi: 10.3389/fpsyg.2020.00470

36. Visted E, Sørensen L, Osnes B, Svendsen JL, Binder P. The association between self-reported difficulties in emotion regulation and heart rate variability : the salient role of not accepting negative emotions. Front Psychol. (2017) 8:328. doi: $10.3389 /$ fpsyg. 2017.00328

37. Aldao A, Mennin DS. Paradoxical cardiovascular effects of implementing adaptive emotion regulation strategies in generalized anxiety disorder. Behav Res Ther. (2012) 50:122-30. doi: 10.1016/j.brat.2011.12.004

38. Kim HG, Cheon EJ, Bai DS, Lee YH, Koo BH. Stress and heart rate variability: a meta-analysis and review of the literature. Psychiatry Investig. (2018) 15:23545. doi: 10.30773/pi.2017.08.17

39. Gaab J, Blättler N, Menzi T, Pabst B, Stoyer S, Ehlert U. Randomized controlled evaluation of the effects of cognitivebehavioral stress management on cortisol responses to acute stress in healthy subjects. Psychoneuroendocrinology. (2003) 28:767-79. doi: 10.1016/S0306-4530(02)00069-0

40. Castro-Rodrigues P, Camacho M, Almeida S, Marinho M, Soares C, BarahonaCorrêa JB, et al. Criterion validity of the yale-brown obsessive-compulsive scale second edition for diagnosis of obsessive-compulsive disorder in adults. Front Psychiatry. (2018) 9:1-10. doi: 10.3389/fpsyt.2018.00431

41. Goodman WK, Price LH, Rasmussen SA, Mazure C, Fleischmann RL, Hill $\mathrm{CL}$, et al. The yale-brown obsessive compulsive scale. I. Development, use, and reliability. Arch Gen Psychiatry. (1989) 46:1006-11.

42. Goodman WK, Price LH, Rasmussen SA, Mazure C, Delgado P, Heninger GR, et al. The yale-brown obsessive compulsive scale. II. Validity. Arch Gen Psychiatry. (1989) 46:1012-6.
43. Gross JJ, John OP. Individual differences in two emotion regulation processes: implications for affect, relationships, and well-being. J Pers Soc Psychol. (2003) 85:348-62. doi: 10.1037/0022-3514.85.2.348

44. Vaz FM, Martins C, Martins EC. Diferenciação emocional e regulação emocional em adultos portugueses. Psicologia. (2014) 22:123. doi: $10.17575 /$ rpsicol.v22i2.350

45. Abramowitz JS, Deacon BJ. Psychometric properties and construct validity of the Obsessive-Compulsive Inventory-Revised: replication and extension with a clinical sample. J Anxiety Disord. (2006) 20:1016-035. doi: 10.1016/j.janxdis.2006.03.001

46. Foa EB, Huppert JD, Leiberg S, Langner R, Kichic R, Hajcak G, et al. the obsessive-compulsive inventory: development and validation of a short version. Psychol Assess. (2002) 14:485-96. doi: 10.1037/1040-3590.14.4.485

47. Trigo M, Canudo N, Branco F, Silva D. Estudo das propriedades psicométricas da Perceived Stress Scale (PSS) na população portuguesa. Psychologica. (2010) 10:353-78. doi: 10.14195/1647-8606_53_17

48. Cohen S, Kamarck T, Mermelstein R. A global measure of perceived stress. $J$ Health Soc Behav. (1983) 24:385. doi: 10.2307/2136404

49. Cohen J. Statistical Power Analysis for the Behavioral Sciences. 2nd ed. New Jersey, NJ: Lawrence Erlbaum Associates (1988).

50. Miles J. Tolerance and variance inflation factor. In: Wiley StatsRef: Statistics Reference Online. Chichester: John Wiley \& Sons, Ltd. p. 1-2.

51. Williams M, Alberto C, Grajales G. Assumptions of multiple regression: correcting two misconceptions - practical assessment, research \& evaluation. Eval Práct Investig Eval. (2013) 18, 1-14. doi: 10.7275/55hn-wk47

52. Baron RM, Kenny DA. The moderator-mediator variable distinction in social psychological research: conceptual, strategic, and statistical considerations. $J$ Pers Soc Psychol. (1986) 51:1173-82. doi: 10.1037/0022-3514.51.6.1173

53. Judd CM, Kenny DA. Data analysis in social psychology: recent and recurring issues. In: Handbook of Social Psychology. Hoboken, NJ: John Wiley \& Sons, Inc. p. 115-39.

54. Kenny DA, Kashy D, Bolger N. Data analysis in social psychology. Handb Soc Psychol. (1998) 1:233-65.

55. Biesanz JC, Falk CF, Savalei V. Assessing mediational models: testing and interval estimation for indirect effects. Multivariate Behav Res. (2010) 45:661701. doi: 10.1080/00273171.2010.498292

56. Preacher KJ, Rucker DD, Hayes AF. Addressing Moderated Mediation Hypotheses: theory, Methods, and Prescriptions. Multivariate Behav Res. (2007) 42:185-227. doi: 10.1080/00273170701341316

57. Hayes AF. Beyond Baron and Kenny: statistical mediation analysis in the new millennium. Commun Monogr. (2009) 76:408-20. doi: 10.1080/03637750903310360

58. Agler R, De Boeck P. On the interpretation and use of mediation: multiple perspectives on mediation analysis. Front Psychol. (2017) 8:111. doi: $10.3389 /$ fpsyg. 2017.01984

59. Milyavsky M, Webber D, Fernandez JR, Kruglanski AW, Goldenberg A, Suri $\mathrm{G}$, et al. To reappraise or not to reappraise? Emotion regulation choice and cognitive energetics. Emotion. (2019) 19:964-81. doi: 10.1037/emo0000498

60. Suri G, Whittaker K, Gross JJ. Launching reappraisal: it's less common than you might think. Emotion. (2015) 15:73-7. doi: 10.1037/emo0000011

61. Ortner CNM, Ste Marie M, Corno D. Cognitive costs of reappraisal depend on both emotional stimulus intensity and individual differences in habitual reappraisal. PLoS ONE. (2016) 11:e0167253. doi: 10.1371/journal.pone. 0167253

62. Moore SA, Zoellner LA, Mollenholt N. Are expressive suppression and cognitive reappraisal associated with stress-related symptoms? Behav Res Ther. (2008) 46:993-1000. doi: 10.1016/j.brat.2008.05.001

63. Troy AS, Shallcross AJ, Brunner A, Friedman R, Jones MC. Cognitive reappraisal and acceptance: effects on emotion, physiology, and perceived cognitive costs. Emotion. (2018) 18:58-74. doi: 10.1037/emo0000371

64. Raposo-Lima C, Morgado P. The role of stress in obsessivecompulsive disorder: a narrative review. Harv Rev Psychiatry. (2020) 28:356-70. doi: 10.1097/HRP.0000000000000274

65. Lithari C, Frantzidis CA, Papadelis C, Vivas AB, Klados MA, Kourtidou-Papadeli $\mathrm{C}$, et al. Are females more responsive to emotional stimuli? A neurophysiological study across arousal and valence dimensions. Brain Topogr. (2010) 23:27-40. doi: 10.1007/s10548-009$0130-5$ 
66. Berke DS, Reidy D, Zeichner A. Masculinity, emotion regulation, and psychopathology: A critical review and integrated model. Clin Psychol Rev. (2018) 66:106-16. doi: 10.1016/j.cpr.2018.01.004

67. Cutuli D. Cognitive reappraisal and expressive suppression strategies role in the emotion regulation: an overview on their modulatory effects and neural correlates. Front Syst Neurosci. (2014) 8:1-6. doi: 10.3389/fnsys.2014.00175

68. Miklósi M, Martos T, Szabó M, Kocsis-Bogár K, Forintos D. Cognitive emotion regulation and stress: a multiple mediation approach. Transl Neurosci. (2014) 5:64-71. doi: 10.2478/s13380-014-0207-9

69. Kinner VL, Het S, Wolf OT. Emotion regulation: exploring the impact of stress and sex. Front Behav Neurosci. (2014) 8:1-8. doi: 10.3389/fnbeh.2014.00397

70. Shermohammed M, Mehta PH, Zhang J, Brandes CM, Chang LJ, Somerville LH. Does psychosocial stress impact cognitive reappraisal? Behav Neural Evid J Cogn Neurosci. (2017) 29:1803-16. doi: 10.1162/jocn_a_01157

71. Tsumura H, Sensaki J, Shimada H. Stress-induced cortisol is associated with generation of non-negative interpretations during cognitive reappraisal. Biopsychosoc Med. (2015) 9:23. doi: 10.1186/s13030-0150049-X

72. Oei NYL, Veer IM, Wolf OT, Spinhoven P, Rombouts SARB, Elzinga BM. Stress shifts brain activation towards ventral 'affective' areas during emotional distraction. Soc Cogn Affect Neurosci. (2012) 7:403-412. doi: 10.1093/scan/nsr024

73. Raio CM, Orederu TA, Palazzolo L, Shurick AA, Phelps EA. Cognitive emotion regulation fails the stress test. Proc Natl Acad Sci USA. (2013) 110:15139-44. doi: 10.1073/pnas.1305706110

74. Quinn ME, Joormann J. Executive control under stress: relation to reappraisal ability and depressive symptoms. Behav Res Ther. (2020) 131:103634. doi: 10.1016/j.brat.2020.103634

75. Kohn N, Eickhoff SB, Scheller M, Laird AR, Fox PT, Habel U. NeuroImage Neural network of cognitive emotion regulation - an
ALE meta-analysis and MACM analysis. Neuroimage. (2014) 87:34555. doi: 10.1016/j.neuroimage.2013.11.001

76. Zator K. Obsessive compulsive disorder as stress and coping: cognitive models and associated treatments. West Undergrad Psychol J. (2014) 2, 777-780.

77. Goodwin GM. The overlap between anxiety, depression, and obsessive-compulsive disorder. Dialogues Clin Neurosci. (2015) 17:249-60. doi: 10.31887/DCNS.2015.17.3/ggoodwin

78. Yap K, Mogan C, Moriarty A, Dowling N, Blair-West S, Gelgec C, et al. Emotion regulation difficulties in obsessive-compulsive disorder. J Clin Psychol. (2018) 74:695-709. doi: 10.1002/jclp.22553

79. Schäfer JÖ, Naumann E, Holmes EA, Tuschen-Caffier B, Samson AC. Emotion regulation strategies in depressive and anxiety symptoms in youth: a meta-analytic review. J Youth Adolesc. (2017) 46:261-76. doi: 10.1007/s10964-016-0585-0

80. Picó-Pérez M, Radua J, Steward T, Menchón JM, Soriano-Mas C. Emotion regulation in mood and anxiety disorders: a meta-analysis of fMRI cognitive reappraisal studies. Prog Neuropsychopharmacol Biol Psychiatry. (2017) 79:96104. doi: 10.1016/j.pnpbp.2017.06.001

Conflict of Interest: The authors declare that the research was conducted in the absence of any commercial or financial relationships that could be construed as a potential conflict of interest.

Copyright (C) 2021 Ferreira, Couto, Sousa, Vieira, Sousa, Picó-Pérez and Morgado. This is an open-access article distributed under the terms of the Creative Commons Attribution License (CC BY). The use, distribution or reproduction in other forums is permitted, provided the original author(s) and the copyright owner(s) are credited and that the original publication in this journal is cited, in accordance with accepted academic practice. No use, distribution or reproduction is permitted which does not comply with these terms. 